Ann. Zootech., I978, 27 (3), 355-36r.

\title{
Méthode rapide de dosage de l'oxyde de chrome dans les aliments, les fèces et les contenus digestifs par titrage après oxydation nitro-perchlorique
}

\author{
E. FRANÇOIS, Nicole THILL (*) et A. THFWIS (**)
}

avec la collaboration technique de A. Colinet $\left(^{*}\right)$, J. C. Pichos (*) et M. Pichon (*)

\author{
Station de Chimie et de Physique agricoles, \\ (*) Station de Zootechnie, \\ Centre de Recherches agronomiques de l'État, \\ 22, avenue de la Faculté d'Agronomie, B 5800 Gembloux (Belgique) \\ (**) Chaire de Physiologie animale et de Zootechnie, \\ Faculté des Sciences agronomiques de l'État, \\ 2, passage des Déportés, B 5800 Gembloux (Belgique)
}

\begin{abstract}
Résumé
Une étude critique des diverses méthodes proposées pour le dosage de l'oxyde de chrome, $\mathrm{Cr}_{2} \mathrm{O}_{3}$, au cours des applications zootechniques de cet indicateur amène les auteurs à proposer une nouvelle technique de détermination ne nécessitant aucun transfert.

La matière organique est détruite et l'oxyde de chrome oxydé par le mélange nitroperchlorique en une seule opération.

Une courte ébullition en présence de persulfate, qui assure l'oxydation complète du chrome, est suivie du titrage direct par le sel de Mohr.

L'emploi de l'appareil proposé par ChaT (1966) pour la destruction perchlorique de la matière organique élimine les risques inhérents à l'emploi de cet acide.

La méthode proposée est précise et très rapide; le titrage peut, en effet, être aisément effectué automatiquement.
\end{abstract}

\section{Introduction}

L'oxyde de chrome, $\mathrm{Cr}_{2} \mathrm{O}_{3}$, marqueur externe de la phase solide des digesta est très utilisé dans des études de digestion. 'THIL, et al. (I978) ont montré qu'il n'est pas absorbé et que, administré sous une forme appropriée (papier à l'oxyde de chrome), son comportement au cours du transit est fort semblable à celui du radiocérium, considéré par de nombreux auteurs comme marqueur idéal (McRAE, I974). L'oxyde de chrome possède donc deux des qualités essentielles d'un bon marqueur (KoTB et LUCKEY, I972). Cependant, la bibliographie montre que les 
résultats des expériences menées avec cet indicateur - détermination du coefficient de digestibilité, évaluation de la production fécale à 1'herbage — ont été souvent décevants (CRAMPTON et LLOYD, I95I; CoRBE'Tr et al., I958; HARdison et al., I959); les échecs ont été attribués à des causes diverses : rétention de 1'indicateur dans le tube digestif, régurgitation d'une partie de la dose administrée, récolte incomplète des fèces et même absorption au travers de la paroi digestive.

Les méthodes de dosage utilisées semblent cependant avoir été assez rarement incriminées explicitement par les expérimentateurs (DAY, I954; ChrIsTraN, WII,IIAMS et CARTER, I965).

Une étude critique des techniques existantes - souvent fort laborieuses nous a permis d'analyser les sources d'erreurs possibles et nous a amenés à élaborer une nouvelle technique de dosage simple, rapide et précise.

\section{Les méthodes existantes}

S'il s'agit d'un composé chimique bien défini, pratiquement insoluble dans l'eau, le nombre considérable de techniques de dosage proposées et les commentaires de leurs utilisateurs (DAY, I954; PETRY et RAPP, I97I) semblent montrer que sa détermination dans les aliments, fèces et contenus digestifs peut poser de nombreux problèmes aux expérimentateurs.

Si la destruction de la matière organique se fait par calcination -- ce qui est le cas pour la plupart des méthodes proposées - une attaque du $\mathrm{Cr}_{2} \mathrm{O}_{3}$ devra lui faire suite.

Les cendres peuvent subir une fusion oxydante alcaline. Les techniques proposées dérivent de celles de Paloheimo (I935) et de Barnicoat (I945) (Schurch, Litoyd et Crampton, i950; DANSKy et Hili, I952; Brisson, I956).

Pour réaliser l'oxydation des cendres, les chercheurs de la Station expérimentale de Hamilton en Nouvelle-Zélande (Coup et LANCASTER, I952) utilisent le chromate en milieu acide sulfurique et phosphorique. Il s'agit d'une méthode laborieuse à laquelle ils apportèrent des modifications successives (CHRISTran et Coup, I954; STEVENSON et DELANGEN, I960).

Mais, en utilisant les acides oxydants, il est possible de réaliser en une étape la destruction de la matière organique et l'oxydation de $\mathrm{Cr}_{2} \mathrm{O}_{3}$. C'est la voie choisie par Bolin, King et Klosterman (I952), Kimura et Mililer (I957), Hili et ANDERSON (I958), WHITBY et LANG (I960), qui emploient l'acide perchlorique en mélange avec l'acide nitrique ou sulfurique en présence de molybdate.

Si la détermination finale du chrome se faisait, au début, volumétriquement (Barnicoat, I945; Kane, Jacobson et MoOre, I950; Coup et Lancaster, I952; Christian et CoUp, I954; WhitBy et LANG, I960), les méthodes plus récentes choisissent généralement la photométrie du chromate, jugée plus rapide (KImurA et MILLER, I957; BRISSON, I956; HiLL et ANDERSON, I958; STEVENSON et DELANGEN, I960; PETRY et RAPP, I97I).

Il est certain que la destruction de la matière organique par la voie humide réalisant l'oxydation de l'indicateur en tme étape est la plus rapide; c'est pourquoi nous l'avons adoptée. Cependant l'emploi d'acide perchlorique est loin d'être sans danger; et par ailleurs, si l'oxydation incomplète du $\mathrm{Cr}_{2} \mathrm{O}_{3}$ constitue une importante source d'erreurs dans les techniques ayant choisi l'oxydation du résidu de calcination en milieu acide (STEVENSON et DELANGEN, I960), le même phénomène a été mis en évidence par Day (I954) pour la méthode de Bolin, KInG et KL,OsterMAN (I952), qui utilise une attaque sulfo-perchlorique. 
Elle a montré que cette technique peut produire des résultats trop faibles par suite de la formation de peroxyde d'hydrogène aux dépens de l'acide perchlorique, peroxyde qui réduirait le $\mathrm{Cr}$ hexavalent. Ajoutons qu'en présence de chlorures - pratiquement toujours présents - d'importantes pertes de chrome hexavalent peuvent se produire par suite de la formation de chlorure de chromyle, $\mathrm{CrO}_{2} \mathrm{Cl}_{2}$, volatil (GORSUCH, I959; HAR'TFORD, I 963 ).

Trois points ont donc retenu notre attention : réduire ou supprimer les risques d'emploi de 1'acide perchlorique, éviter une perte de chrome tout en réalisant l'oxydation complète du chrome en $\mathrm{Cr}^{6+}$. Leur réalisation nous a amenés à élaborer une nouvelle technique de dosage rapide dont l'exposé constitue l'objet du présent article.

\section{Élaboration de la technique de dosage et justification des conditions opératoires}

\section{Destruction de la matière organique et attaque de l'oxyde de chrome}

L'appareil de minéralisation proposé,par CHAT (I966) permet de satisfaire aux deux premières exigences. Il s'agit d'un appareil simple, en verre, permettant de réaliser l'attaque dans un système à reflux comportant un piège. L'emploi de chauffe-ballons (heating mantles) thermostatés recommandés par l'auteur permet le réglage aisé de l'allure de chauffe. Deux légères modifications cependant; d'une part, 1'expérience acquise au cours de dizaines de milliers de minéralisations sans problèmes nous a permis d'éliminer 1'acide sulfurique, et de lui substituer le mélange nitro-perchlorique (2:I); d'autre part, les dimensions du ballon et du piège ont été modifiées en vue du dosage de $\mathrm{Cr}_{2} \mathrm{O}_{3}$ (fig. I). Un plus grand diamètre du col, du ballon et de la tubulure latérale augmente le reflux et diminue la vitesse linéaire des gaz et aérosols produits au cours des réactions d'oxydation assez actives se produisant surtout lors de l'attaque perchlorique des matières organiques ayant résisté à l'acide nitrique; cette dernière ne se produit que lorsque la concentration. de l'acide et sa température ont atteint un seuil donné, par départ de l'eau et de l'acide nitrique excédentaire.

On repère facilement l'allure de chauffe de chaque appareil assurant tne condensation des vapeurs d'acide perchlorique dans la tubulure latérale et on conduit toutes les minéralisations subséquentes, dès le début, à cette allure; on continue l'ébullition pendant une à deux minutes après la solubilisation et le virage de l'oxyde de chrome qui ne se produit qu'après l'oxydation perchlorique des matières organiques résistantes.

Dans ces conditions, aucune perte de chrome par volatilisation de $\mathrm{CrO}_{2} \mathrm{Cl}_{2}$ n'est à craindre. Toute l'opération dure à peu près $30 \mathrm{mn}$ et ne requiert pratiquement pas de surveillance.

L'appareil de ChAT, utilisé dans les conditions de chauffage décrites, évite la dessiccation des sels minéraux, en supprimant toute perte d'acide perchlorique.

Les risques d'explosion des appareils sont extrêmement réduits. I) plus, les conduits d'évacuation de l'air des sorbonnes ne sont pas contaminés par l'acide perchlorique. On élimine ainsi le danger majeur qu'entraîne l'emploi de cet acide : les incendies ou les explosions des dépôts accumulés de longue date et imbibés de condensat.

Le piège est simplement destiné à retenir l'acide nitrique excédentaire. On élimine de la sorte une source importante de pollution de l'air et de dégradation du matériel.

Un contrôle de cette première étape de l'analyse a été réalisé par l'emploi 


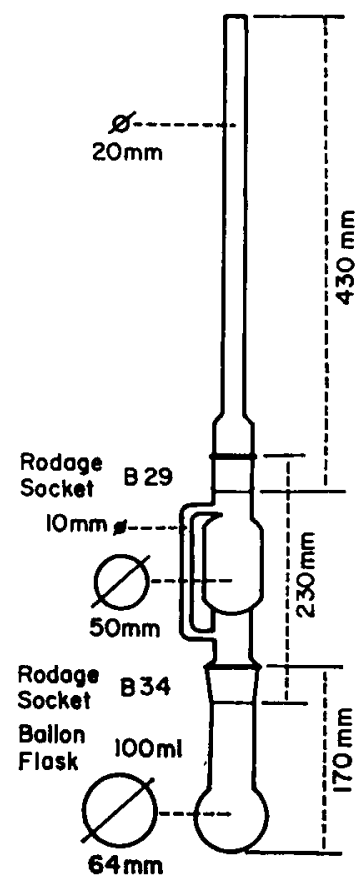

FIG. I. - Appareil de minéralisation suivant Chat (1966), proportions modifiées. Digestion apparatus (CHAT, 1966), modified.

de radiochrome, ${ }^{51} \mathrm{Cr}$; une quantité connue de ce radioisotope ajoutée à un échantillon de matière organique contenant $\mathrm{du} \mathrm{Cr}_{2} \mathrm{O}_{3}$ se retrouve intégralement dans le ballon à la fin de l'attaque. En revanche, si on utilise des ballons à col étroit et si l'on surchauffe, on peut provoquer des pertes énormes.

Le bilan de l'isotope montre que pratiquement tout le chrome volatilisé (99 p. Ioo dans les conditions du test) se retrouve dans le piège.

\section{Réalisation d'une oxydation complète du chrome}

Si tout le $\mathrm{Cr}_{2} \mathrm{O}_{3}$ est décomposé, ce qui est visible, tout le chrome ne se retrouve cependant pas au niveau hexavalent, même en l'absence d'acide sulfurique, si on refroidit rapidement le résidu de minéralisation comme le préconise DAy (I954). Selon cet auteur la présence de peroxydes - formés, selon elle, par l'action déshydratante de l'acide sulfurique sur 1'acide perchlorique - provoquerait une réduction de $\mathrm{Cr}^{6+}$ (Smith, I935). L'apparition graduelle - en quelques heures - de la couleur verte typique des ions $\mathrm{Cr}^{3+}$ dans les résidus acides refroidis semble confirmer cette explication. Le titrage direct au sel de Mohr du chrome dans le résidu de minéralisation donne des résultats complètement aberrants. L'erreur par défaut commise, par suite de la réduction du $\mathrm{Cr}^{6+}$ augmente avec le temps et peut atteindre $20 \mathrm{p}$. Ioo après 24 heures.

Une étape de réoxydation s'avérait donc nécessaire avant la détermination du chrome. Le persulfate, en milieu acide, en présence d'argent, nous a donné d'excellents résultats (SANDeli, I959; Charlot, I96I; HartFord, I963).

L,a détermination du chrome s'effectue simplement, dans le même ballon, 
par titrage direct classique au sel de Mohr, en présence de diméthylamine sulfonée.

PETRY et KAPP (I97I), après une attaque par voie humide suivant BoLIN, KING et KI,OSTERMAN (I952), obtiennent de bons résultats en déterminant en milieu alcalin le chrome par photométrie du chromate, seul ion présent à un $\mathrm{pH}$ supérieur à II. Cette technique requiert cependant des transferts de solution, une centrifugation et deux lavages du précipité abondant se formant à ce $\mathrm{pH}$.

Nous avons préféré la voie titrimétrique qui évite tout transfert du composé à doser et élimine ainsi une source d'erreurs en limitant les manipulations.

\section{La méthode de détermination de l'oxyde de chrome}

\section{Réactifs}

- Mélange de minéralisation $\mathrm{HNO}_{3}$ à 65 p. Ioo p.a. 2 volumes, $\mathrm{HClO}_{4}$ à 70 p. Ioo p.a. I volume.

- Solution à Io p. I Oo de persulfate d'ammonium : $\left(\mathrm{NH}_{4}\right)_{2} \mathrm{~S}_{2} \mathrm{O}_{8}$ p.a.

- Solution nitrique de nitrate d'argent à $\mathrm{I}, 5 \mathrm{p}$. Ioo :

On dissout $\mathrm{I}_{5} \mathrm{~g}$ de $\mathrm{AgNO}_{3}$ p.a. dans de l'eau distillée, on transvase dans un ballon jaugé de $\mathrm{I}$ ooo $\mathrm{ml}$ et on ajoute $35 \mathrm{ml} \mathrm{HNO}_{3}$ à 65 p. IoO p.a. avant de porter au trait avec de l'eau distillée.

- Acide sulfurique au $\mathbf{I} / 6$.

- Acide phosphorique : $\mathrm{H}_{3} \mathrm{PO}_{4}$ conc. p.a. à 65 p. Ioo.

- Solution de diméthylamine sulfonée : à $0,5 \mathrm{p}$. Ioo dans l'eau.

- Solution de bichromate $0,02 \mathrm{n}$ :

Les cristaux de $\mathrm{K}_{2} \mathrm{Cr}_{2} \mathrm{O}_{7}$ p.a. sont broyés au mortier, séchés à $120-\mathrm{r}_{30}{ }^{\circ} \mathrm{C}$ et conservés en exsiccateur. La solution $0,02 \mathrm{n}$ contient $0,98075 \mathrm{~g}$ de bichromate par litre.

- Solution de sel de Moht : Fe $\mathrm{SO}_{4} \cdot\left(\mathrm{NH}_{4}\right)_{2} \mathrm{SO}_{4} \cdot 6 \mathrm{H}_{2} \mathrm{O}$ p.a.

On dissout $7,84 \mathrm{~g}$ de sel dans l'eau distillée transférée dans un ballon de r ooo $\mathrm{ml}$, on y ajoute $135 \mathrm{ml}$ d'acide sulfurique concentré avant de porter au trait. Cette solution est titrée par la solution de $\mathrm{K}_{2} \mathrm{Cr}_{2} \mathrm{O} 0,02 \mathrm{n}$.

\section{Mode opératoire}

I $\mathrm{g}$ d'échantillon (contenant de 5 à $\mathrm{I}_{5} \mathrm{mg} \mathrm{Cr}_{2} \mathrm{O}_{3}$ ) est transféré dans le ballon de minéralisation et additionné de $25 \mathrm{ml}$ de mélange nitroperchlorique. On assemble 1'appareil de Chat et commence immédiatement 1'attaque. Les échantillons qui ont tendance à mousser sont retirés pendant quelques instants des nids chauffants; ils ne posent plus de problèmes par la suite. Chauffer $2 \mathrm{mn}$ après l'oxydation (virage à 1'orange) de $\mathrm{Cr}_{2} \mathrm{O}_{3}$. L'ensemble de l'opération prend de 20 à $25 \mathrm{mn}$. Une fois refroidi, le résidu de minéralisation est additionné de $50 \mathrm{ml}$ de persulfate à Io p. Ioo et de $I 5 \mathrm{ml}$ de nitrate d'argent à $I, 5 \mathrm{p}$. Ioo. On chauffe à l'ébullition Io mn afin de détruire 1'excès de persulfate. Après refroidissement, on acidifie avec ro $\mathrm{ml}$ d'acide sulfurique approximativement $6 \mathrm{n}(\mathrm{I} / 6)$, ajoute $5 \mathrm{ml}$ d'acide phosphorique concentré, 3 gouttes de diméthylamine sulfonée et titre au sel de Mohr. Le virage est très facilement perçu.

Si $v$ est le volume de solution employé en $\mathrm{ml}, t$ son titre, $p$ la prise d'essai en $\mathrm{g}$ et 0,02533667 le milliéquivalent-gramme de $\mathrm{Cr}_{2} \mathrm{O}_{3}$, la concentration de $\mathrm{Cr}_{2} \mathrm{O}_{3}$ dans la matière est donnée par :

$$
\mathrm{C}=\frac{v \cdot t \cdot 0,02533667}{p}
$$




\section{Justification des conditions opératoires}

La méthode présentée est plus rapide que les méthodes décrites antérieurement et d'un emploi très simple; c'est, à notre avis, un avantage considérable, les risques d'erreurs inhérents aux solutions adoptées ont été éliminés.

La méthode est rapide et se prête bien au travail en série, car on a évité tout transfert. La titrimétrie de la solution trouble a été préférée pour cette raison. Le titrage final peut être aisément automatisé. Une électrode combinée (platinecalomel) peut être plongée directement dans le ballon contenant la solution ayant subi l'oxydation au persulfate. Les appareils nécessaires relativement peu cồteux, sont fort répandus dans les laboratoires analytiques.

Dans ces conditions, et pour autant que l'on dispose d'un matériel de chauffage suffisant, deux douzaines de "nids chauffants ", deux opérateurs pourront traiter I5o échantillons par jour.

Le titrage pourrait éventuellement être remplacé par la détermination colorimétrique du chrome. L'automatisation de cette dernière étape (MATHIEson, I970) pourrait compenser la perte de temps qu'entraînent la mise au volume de la solution et les transferts. Il faut noter que les appareils nécessaires à 1'automatisation sont généralement assez cồteux, dans ce cas.

Le $\mathrm{Cr}_{2} \mathrm{O}_{3}$ n'est pas une substance-type; nous avons néanmoins contrôlé la dissolution et l'oxydation d'une dose de $20 \mathrm{mg}$ de $\mathrm{Cr}_{2} \mathrm{O}_{3}$ (qualité pour analyse) ajoutée à de la cellulose pure : I02,25 p. Ioo $\pm 0,60(\mathrm{P}=0,95)$.

La récupération d'une quantité connue de $\mathrm{K}_{2} \mathrm{Cr}_{2} \mathrm{O}_{7}$ o,02 n ajoutée à de la cellulose pure a été de $99,20 \pm 0,4 \mathrm{I}(\mathrm{P}=0,95)$. Si on ajoute cette dose à des fèces broyées, on obtient généralement une valeur légèrement supétieure à roo $\mathrm{p}$. Ioo (I-2 p. IOO), đue à la présence de faibles quantités de manganèse.

Cette interférence, qui ne nous a jamais gênés, peut être éliminée aisément.

Si on étudie par exemple la courbe d'excrétion fécale d'une dose de $\mathrm{Cr}_{2} \mathrm{O}_{3}$ destinée à caractériser le trarısit digestif, la valeur du blanc sera obtenue sur les fèces émises avant administration de cette dose.

Accepté pour publication en mai 1978.

\section{Summary}

A rapid volumetric method for the determination of chromium sesquioxide in feeds, faeces and digesta after nitroperchloric digestion

A critical bibliographical study induced the authors to present a new technique for the determination of chromium sesquioxide in samples used in digestibility experiments. Organic matter is destroyed and chromium sesquioxide oxidized by a mixture of nitric and perchloric acids in a single operation. After a short ebullition with persulfate necessary to bring all chromium to the hexavalent state, the latter is directly titrated with Mohr's salt.

The proposed method is very rapid and precise; the manipulations are reduced to the strict minimum. Furthermore, use of the glass apparatus proposed by CHAT ( 1966 ) completely eliminates the risks resulting from the use of perchloric acid.

\section{Références bibliographiques}

BARNICOAT C. R., I945. Estimation of apparent digestibility coefficients by means of an inert "reference substance". N. Z. Jl. Sci. Technol., 27, 202-212.

Bolin D. W., KING R. P., KLosterman F. W., i952. A simplified method for the determination of chromic oxide $\left(\mathrm{Cr}_{2} \mathrm{O}_{3}\right)$ when used as an index substance. Science, 116, 634-635. 
Brisson G. J., 1956. On the routine determination of chromic oxide in feces. Can. J. Agric, Sci, 36, 210-2I2.

Chariot G., I961. Les méthodes de la chimie analytique, $4^{\mathrm{e}}$ édition, 7 or. Masson et Cie, Paris VI".

Chat G., I966. Nouvelle méthode de minéralisation des végétaux pour les analyses chimiques. C. R. hebd. Séanc. Acad. Agric. Fr., 51, г087-1093.

Ciristian K. R., CovP M. R., r954. Measurement of feed intake by grazing cattle and sheep. VI. The determination of chromic oxide in faeces. N. Z. Jl. Sci. Technol., 36, 328-33o.

Christian K. R., Williams V. J., CarTer A. H., I965. The estimation of faeces output of stallfed sheep using chromic oxide marker. N. Z. Jl. Agric. Res., 8, 530-54I.

Corbett J. L., Greenhalgh J. F. D., Gwynn P. E., Wal ker D., i958. Excretion of chromium sesquioxide and polyethylene glycol by dairy cows. Br. J. Nutr., 12, 266-276.

COUP M. R., LANCASTER R. J., 1952. The measurement of feed intake by grazing cattle and sheep. Part II. The determination of chromic oxide and monastral blue in cow faeces. $N$. Z. Jl. Sci Technol., 34 347-353.

Crampton E. W., I LOYd L. E., 195I. Studies with sheep on the use of chromic oxide as an index of digestibility of ruminant rations. J. Nutr., 45, 319-327.

DaNsky L. M., HILL F. W., I952. Application of the chromic oxide indicator method to balance studies with growing chickens. J. Nutr., 47, 449-459.

DAY K. M., I954. Source of error in determination of chromic oxide using perchloric-sulfuric acid digestion method. Science, 120, 717-718.

GoRsuch T. T., I959. Radiochemical investigations on the recovery for analysis of trace elements. in inorganic and biological material. Analyst, 84, I35-I73.

Hardison W. A., I IN Kous W. N., ENGEL R. W., I959. Observation on the use of $\mathrm{Cr}_{2} \mathrm{O}_{3}$ for estimating the feeal output of dairy animals. J. Dairy Sci., 42, 346-352.

HARTFORd W. H., 1963. Chromium. In KOLTHOFN I. M., Elving P. J., Treatise on analytical chemistry. Part II, vol. 8, 273-377, Interscience publishers New York, London, Sydney.

HILL F. W., ANJERSON D. L., I958. Comparison of metabolizable energy and productive energy determination with growing chicks. J. Nutr., 64, 587-602.

Kane E. A., JAcobson W. C., MoOrE L. A., I950. A comparison of techniques used in digestibility studies with dairy cattle. $J$. Nutr., 41, 583-596.

KIMURA F. T., MILLER V. L., I957. Improved determination of chromic oxide in cow feed and feces. J. Agr. Fd. Chem., 5, 216.

Kотв A. R., I.ckity T. D., I972. Markers in nutrition. Nutr. Abstr. Rev., 42, 81 3-845.

MACRAE J. C., 1974. The use of intestinal markers to measure digestive function in ruminants. Proc. Nutr. Soc., 33, I 47 -I 54.

Mathieson J., I970. The automated estimation of chromic oxide, Proc. Nuir. Soc, 29, 30A-3 IA.

Palohieimo L., PaIohfimo I., I935. Fine photometrische Methode zur Bestimmung der $\mathrm{Cr}_{2} \mathrm{O}_{3}$ bei Verdaulichkeitsuntersuchungen nach sog. quantitativen Indikatorverfahren. Tievernährung, 7, 3I 7-324.

Petry H., RAPY W., 197r. Zur Problematik der Chromoxidbestimmung in Verdauungsversuchen. Z. Tierphysiol. Tievernähr. Futtermittolk, 27, $181-189$.

SANDEL, E. B., I959. Colorimetric determination of traces of metals, 396. 3 rd edition. Interscience Publishers, New York, London.

Schürch A. F., Lloyd L. E., Crampton E. W., I950. The use of chromic oxide as an index for determining the digestibility of a diet. $J$. Nutr., 41, $629-636$.

SMITH G. F., 1935. Mixed perchloric, sulluric and phosphoric acids and their application in anctlysis. First ed., G. Frederick Smith Chemical Co., Columbia, Ohio, U.S.A.

Stevfinson A. E., Delangen H., i960. Measurement of feed intake by grazing cattle and sheep. VI. Modified wet digestion method for determination of chromic oxide in faeces. $N, Z . J /$. Agric. Res., 3, 314-319.

Thili N., Fraxcois E., Thewis A., Thimlfmaxs M. F., 1978 . Comparaison, chez le moutoñ de l'oxyde de chrome-tapier et du radiocérium, marqueurs de la phase solide des digesta. Ann. Zootech., 27, 363-376.

Whitby L. G., LANG D. J., Ig60. Experience with the chromic oxide method of fecal marking in metabolic balance investigation on human. $J$. Clin. Invest., 39, 854-863. 